\title{
Introduction to the Summary of Notifiable Noninfectious Conditions and Disease Outbreaks - United States
}

\author{
Ralph J. Coates, $\mathrm{PhD}^{1}$ \\ Martha Stanbury, $\mathrm{MSPH}^{2}$ \\ Ruth Jajosky, DMD ${ }^{1}$ \\ Kimberly Thomas, $\mathrm{MPH}^{1}$ \\ Michele Monti, $\mathrm{MS}^{3}$ \\ Patricia Schleiff, MS 4 \\ Simple D. Singh, MD 5 \\ ${ }^{1}$ Division of Health Informatics and Surveillance, Center for Surveillance, Epidemiology, and Laboratory Services, CDC \\ ${ }^{2}$ Division of Environmental Health, Michigan Department of Health and Human Services, Lansing, Michigan \\ ${ }^{3}$ Division of Environmental Hazards and Health Effects, National Center for Environmental Health, CDC \\ ${ }^{4}$ Respiratory Health Division, National Institute for Occupational Safety and Health, CDC \\ ${ }^{5}$ Division of Cancer Prevention and Control, National Center for Chronic Disease Prevention and Health Promotion, CDC
}

With this 2016 Summary of Notifiable Noninfectious Conditions and Disease Outbreaks — United States, CDC is publishing official statistics for the occurrence of nationally notifiable noninfectious conditions and disease outbreaks for the second time in the same volume of $M M W \mathrm{R}$ as the annual Summary of Notifiable Infectious Diseases and Conditions (1). As was the case for the 2015 Summary of Notifiable Noninfectious Conditions and Disease Outbreaks (2), this joint publication is the result of a request by the Council of State and Territorial Epidemiologists (CSTE) to provide readers with information on all nationally notifiable conditions and disease outbreaks in a single publication.

The 2016 Summary of Notifiable Noninfectious Conditions and Disease Outbreaks includes for the first time a chapter on acute pesticide-related illness and injury from nonoccupational pesticide exposure whereas the 2015 Summary of Notifiable Noninfectious Conditions and Disease Outbreaks included only acute pesticiderelated illness and injury from occupational exposure (2). This summary includes seven chapters addressing the following subjects: acute pesticide-related illness and injury arising from occupational exposure (3), acute nonoccupational pesticide-related illness and injury (4), cancer (5), elevated blood lead levels among children ( $)$, elevated blood lead levels among adults ( () , silicosis (8), and foodborne and waterborne disease outbreaks (9).

Information on elevated lead exposure is provided in two separate chapters $(6,7)$ because the principal source of elevated blood lead levels in children (lead paint in homes) is different from the principal source in adults (lead exposure at work). Responsibilities for monitoring blood lead levels are assigned to different Centers at CDC. The National Center

Corresponding author: Kimberly Thomas, Division of Health Informatics and Surveillance, Center for Surveillance, Epidemiology, and Laboratory Services, CDC. Telephone: 404-498-0282; E-mail: kit9@cdc.gov. for Environmental Health (NCEH) has primary responsibility for preventing disease from environmental hazards (principally nonoccupational) and manages the Childhood Blood Lead Surveillance (CBLS) system (7). The National Institute for Occupational Safety and Health (NIOSH) is responsible for preventing disease from workplace hazards and manages the Adult Blood Lead Epidemiology and Surveillance (ABLES) system (G). Information on acute pesticide-related illness and injury also is provided in separate chapters for occupational and nonoccupational exposure $(3,4)$. NIOSH led preparation of the chapter on acute occupational pesticide-related illness and injury (3), and NCEH led preparation of the chapter on acute nonoccupational pesticide-related illness and injury (4). NCEH obtained data on acute nonoccupational pesticide related illness from the NIOSH Sentinel Event Notification System for Occupational Risks (SENSOR)-Pesticides program, which collects data from some states on both occupational and nonoccupational cases.

Each of the seven chapters in this summary presents the most recent statistics available to the applicable CDC program. Local, state, and territorial health departments and other agencies in their jurisdictions (e.g., departments of labor, environmental protection agencies, cancer registries, and their agents) submit data to the National Center for Chronic Disease Prevention and Health Promotion (NCCDPHP), the National Center for Emerging and Zoonotic Infectious Diseases (NCEZID), NCEH, and NIOSH.

CDC's Center for Surveillance, Epidemiology, and Laboratory Services (CSELS) coordinated the development and publication of this annual summary. Comments and suggestions from readers on this new combined publication are encouraged, including ones about whether the information presented could be made more useful. Comments should be sent to the following e-mail account: NNDSSweb@cdc.gov, and display the term "Noninfectious Disease MMWR Report" in the subject line. 


\section{Background}

As with nationally notifiable infectious diseases, to prevent and control nationally notifiable noninfectious conditions and disease outbreaks, public health authorities require regular, frequent, and timely information. A brief history of the reporting of nationally notifiable conditions in the United States is available at http://wwwn.cdc.gov/nndss/history. html. In 1961, responsibility for collecting data on nationally notifiable diseases and deaths in 122 U.S. cities was transferred from the National Office of Vital Statistics to CDC.

CDC's collection of data on nationally notifiable noninfectious conditions and disease outbreaks is based primarily on surveillance conducted at the local, state, and territorial levels by health departments and other agencies on reportable conditions in each jurisdiction. Legislation, regulation, or other rules in those jurisdictions require health care providers, hospitals, laboratories, and others to provide information on reportable conditions to public health authorities or their agents. The list of reportable conditions in each jurisdiction varies over time and across jurisdictions. More information is available at http://www.cste.org/?SRCA. Public health surveillance of noninfectious conditions and disease outbreaks at the local, state, and territorial levels protects the public's health by ensuring the proper identification of conditions and health hazards. Public health officials use these data to monitor trends in these conditions, identify populations or geographic areas at high risk, plan prevention and control policies and other interventions, allocate resources effectively, coordinate activities, and assess the effectiveness of their efforts. Local, state, and territorial health departments also use these data to assist the federal government in meeting requirements under the International Health Regulations to identify, respond to, and share information about adverse health events that might constitute a Public Health Emergency of International Concern (PHEIC) (http://www.cdc.gov/ globalhealth/ihr). Health departments have agreed to report information about a potential PHEIC to federal agencies (including $\mathrm{CDC}$ ) that have primary responsibility at the national level for monitoring such events. After evaluating whether an event constitutes a potential PHEIC, CDC notifies the U.S. Department of Health and Human Services (HHS). HHS, after further evaluation, reports potential PHEICs to the World Health Organization (WHO), which might declare the event a PHEIC. More detailed information on this process is found in the Summary of Notifiable Infections Diseases and Conditions (1) and from WHO (http://apps.who.int/iris/ bitstream/10665/43883/1/9789241580410_eng.pdf).

A selected set of reportable conditions is designated as nationally notifiable. For most of these conditions, notifications are submitted to CDC by state, local, and territorial health departments. Public health officials at state, local, and territorial health departments and CDC collaborate in identifying conditions to consider for national notification. CSTE, in consultation with CDC, recommends revisions to the list of nationally notifiable conditions. Conditions are added to the list as new pathogens, environmental hazards, or conditions emerge as public health concerns. Conditions are deleted when surveillance is not found to be useful. Current and historic lists of nationally notifiable conditions and definitions used for classifying and counting cases consistently at the national level across jurisdictions are available at http://wwwn.cdc.gov/nndss. CDC uses these data to monitor trends at the national level, develop and implement programs, allocate resources, and assess the effectiveness of national efforts at prevention and control.

Reporting of conditions at the local, state, and territorial levels is mandated by legislation or regulations at those levels, and submission of notifications to CDC is voluntary. Underreporting of noninfectious conditions and disease outbreaks to local and state health departments occurs, and completeness of reporting and therefore of notification to CDC varies by condition (3-15).

Although the sources of data for nationally notifiable infectious diseases and nationally notifiable noninfectious conditions and disease outbreaks are the same (i.e., local, state, and territorial jurisdictions' data on reportable conditions) and the purpose is the same (i.e., monitoring and responding to the condition to improve population health), there are a number of variations and differences among the conditions in this annual summary (1,3-8) (https://wwwn.cdc.gov/nndss/conditions/). Case-based surveillance of such nationally notifiable conditions as acute pesticide-related illness or injury, silicosis, and cancer is focused on detecting persons who have a condition that meets the criteria specified in national disease-specific case definitions and on collecting information about those persons' conditions. In contrast, surveillance of outbreaks of foodborne and waterborne illness seeks to identify clusters of sick persons with a common exposure (as opposed to persons with a specific disease). Foodborne disease outbreaks are defined as two or more cases of similar illness resulting from common ingestion of a food. Waterborne disease outbreaks are defined as two or more cases of a similar illness resulting from common exposure to water or water-associated chemicals. Information is collected about the characteristics of the disease outbreaks, including data from epidemiologic and environmental investigations. Even among conditions for which case-based surveillance methods are used, substantial variation exists regarding the meaning of a condition. For example, for a condition such as elevated blood lead levels, surveillance identifies persons who have been exposed to a hazard on the basis of a laboratory test. 
In contrast, for many other conditions, a diagnosis based on clinical and/or pathological criteria is needed to meet the case definition for a notification to CDC. This variability makes it challenging for readers to compare statistics easily across conditions and geographic locations.

The meaning of the date of the occurrence of the condition varies among the conditions. For infectious diseases, the meaning of the date varies across jurisdictions as well as by condition and might be a date of symptom or disease onset, diagnosis, or laboratory result; date of death; date the case was reported to a jurisdiction or date the case notification was sent to CDC; or date the criteria in the national surveillance case definition were met (http://wwwn.cdc.gov/nndss/ document/MMWR_Week_overview.pdf). For cancer, as for some infectious diseases (including the arboviral diseases, tuberculosis, and human immunodeficiency virus infection diagnosis), the date of occurrence is the date the condition was diagnosed. For silicosis, the date of occurrence is the date of the initial report (e.g., the date of a hospital discharge report, clinician report, or a workers' compensation claim). For lead screening test results, the date of occurrence is the date of a test. For disease outbreaks, the date of occurrence is the date of the illness onset of the first person with a case in the outbreak.

The source and definitions of race and ethnicity also vary over time and among conditions. For example, information about race and ethnicity for lead exposure is based on selfreport, whereas for cancer incidence, such information is based on medical records, which might or might not be based on self-report, or comes from matching the names of persons with cancer with lists of surnames for different ethnic groups or with tribal registries. For silicosis, race and ethnicity are based on selfreport, report from next-of-kin, or information from medical records. Race- and ethnicity-specific information among the conditions also might vary depending on differences in the jurisdictions' systems for submitting notifications to CDC and the need to protect confidentiality of private health information.

The chapters in this summary use U.S. Census Bureau data sets for the denominators in the rate estimates. However, there is variation across the chapters in which specific U.S. Census Bureau data sets are used.

There are additional notable differences among the chapters in this annual summary concerning the criteria used by CDC programs to determine which case notifications are summarized and published in $M M W R$ (i.e., publication criteria). For data on both infectious and noninfectious conditions submitted to CDC from the jurisdictions, the condition or disease must have been designated as a reportable condition in that jurisdiction for the year of notification to CDC. However, CDC publishes information on foodborne and waterborne disease outbreaks in this annual summary even if the condition was not on the jurisdiction's reportable conditions list. Additional criteria, based on characteristics that define the conditions and disease outbreaks (http://wwwn.cdc.gov/nndss/conditions), are used in making a final determination on publication in this annual summary (Box).

BOX. Criteria defining nationally notifiable conditions and disease outbreaks used to determine whether notifications to CDC are published in the annual Summary of Notifiable Noninfectious Conditions and Disease Outbreaks

\section{Condition/Outbreak}

Acute nonoccupational pesticide-related illness

Acute occupational pesticide-related illness

Cancer

Lead exposure test results in children

Lead exposure test results in adults

Silicosis

Foodborne disease outbreak

Waterborne disease outbreak

\section{Classification}

Definite, probable, possible, and suspicious

Definite, probable, possible, and suspicious

Confirmed

Confirmed

Confirmed

Confirmed

Two or more cases of a similar illness resulting from the ingestion of the same food

Two or more cases of a similar illness linked epidemiologically by time and location to exposure to water or waterassociated chemicals volatized into the air 


\section{Data Sources}

Final data for nationally notifiable noninfectious conditions and disease outbreaks are derived from the surveillance systems of the CDC Centers listed below. Requests for further information regarding these data should be directed to the appropriate Division or program:

- National Center for Chronic Disease Prevention and Health Promotion

- Division of Cancer Prevention and Control

o National Program of Cancer Registries (cancer)

- National Center for Emerging and Zoonotic Infectious Diseases

- Division of Foodborne, Waterborne, and Environmental Diseases

o Foodborne Disease Outbreak Surveillance System (foodborne disease outbreaks)

o Waterborne Disease and Outbreak Surveillance System (waterborne disease outbreaks)

- National Center for Environmental Health

- Division of Environmental Hazards and Health Effects

o Environmental Public Health Tracking Program (acute nonoccupational pesticide-related illness) (from NIOSH Sentinel Event Notification System for Occupational Risks (SENSOR)-Pesticides Program and California Department of Pesticide Regulation)

- Division of Emergency and Environmental Health Services

o Childhood Blood Lead Surveillance (lead exposure test results in children)

- National Institute for Occupational Safety and Health

- Division of Surveillance, Hazard Evaluations, and Field Studies

o SENSOR-Pesticides Program (acute occupational pesticide-related illness)

o Adult Blood Lead Epidemiology and Surveillance (ABLES) Program (lead exposure test results in adults)

- Respiratory Health Division

o State-Based Silicosis Surveillance (silicosis)

\section{References}

1. CDC. Summary of notifiable infectious diseases and conditions-United States, 2014. MMWR Morb Mortal Wkly Rep 2014;63(54).

2. CDC. Summary of notifiable noninfectious conditions and disease outbreaks-United States. MMWR Morb Mortal Wkly Rep 2013;62(54).

3. Calvert GM, Orielb M, Beckmanc J, et al. Acute occupational pesticiderelated illness and injury-United States, 2007-2011. In: CDC. Summary of notifiable noninfectious conditions and disease outbreaksUnited States. MMWR Morb Mortal Wkly Rep 2014;63(55):11-6.

4. Namulanda G, Monti M, Prakash M, et al. Acute nonoccupational pesticide-related illness and injury-United States, 2007-2011. In: CDC. Summary of notifiable noninfectious conditions and disease outbreaksUnited States. MMWR Morb Mortal Wkly Rep 2014;63(55):5-10.

5. Singh SD, Henley SD, Ryerson B. Surveillance for cancer incidence and mortality-United States, 2012. In: CDC. Summary of notifiable noninfectious conditions and disease outbreaks — United States. MMWR Morb Mortal Wkly Rep 2014;63(55):17-58.

6. Raymond J, Brown MJ. Childhood lead exposure-United States, 2007-2013. In: CDC. Summary of notifiable noninfectious conditions and disease outbreaks-United States. MMWR Morb Mortal Wkly Rep 2014;63(55):59-65.

7. Alarcon W, state Adult Blood Lead Epidemiology and Surveillance (ABLES) program investigators. Elevated blood lead levels among adults-United States, 1994-2013. In: CDC. Summary of notifiable noninfectious conditions and disease outbreaks_-United States. MMWR Morb Mortal Wkly Rep 2014;63(55):66-72.

8. Schleiff P, Mazurek J, Reilly MJ, et al. Surveillance for silicosisMichigan and New Jersey, 2003-2011. In: CDC. Summary of notifiable noninfectious conditions and disease outbreaks-United States. MMWR Morb Mortal Wkly Rep 2014;63(55):73-8.

9. Dewey-Mattia D, Roberts VA, Vieira A, Fullerton KE. Foodborne (1973-2013) and waterborne (1971-2013) outbreaks. In: CDC. Summary of notifiable noninfectious conditions and disease outbreaksUnited States. MMWR Morb Mortal Wkly Rep 2014;63(55):78-83.

10. Calvert GM, Karnik J, Mehler L, et al. Acute pesticide poisoning among agricultural workers in the United States, 1998-2005. Am J Ind Med 2008;51:883-98. http://dx.doi.org/10.1002/ajim.20623

11. US Cancer Statistics Working Group. United States cancer statistics: 1999-2012 incidence and mortality web-based report. Atlanta, GA: US Department of Health and Human Services, CDC, National Cancer Institute; 2015.

12. Meyer PA, Pivetz T, Dignam TA, Homa DM, Schoonover J, Brody D. Surveillance for elevated blood lead levels among children-United States, 1997-2001. MMWR Surveill Summ 2003;52(No. SS-10).

13. CDC. Very high blood lead levels among adults-United States, 2002-2011. MMWR Morb Mortal Wkly Rep 2013;62:967-71.

14. Rosenman KD, Reilly MJ, Henneberger PK. Estimating the total number of newly-recognized silicosis cases in the United States. Am J Ind Med 2003;44:141-7. http://dx.doi.org/10.1002/ajim.10243

15. Gould LH, Walsh KA, Vieira AR, et al. Surveillance for foodborne disease outbreaks-United States, 1998-2008. MMWR Surveill Summ 2013;62(No. SS-2). 